\title{
Effect of Curing Method of a Dual-cure Resin Cement on Monkey Pulpal Reaction after Bonding of Tooth-colored Inlay
}

\author{
Yasushi SHIMADA ${ }^{1}$, Md Akhtar UZZAMAN ${ }^{1}$, Junji TAGAMI ${ }^{1,2}$, Toru TANAKA ${ }^{3}$, Takashi NAKATA ${ }^{3}$, \\ Yasuko $\mathrm{NAKAOKI}^{3}$ and Hidehiko $\mathrm{SANO}^{3}$ \\ ${ }^{1}$ Cariology and Operative Dentistry, Department of Restorative Sciences, Graduate School, Tokyo Medical and Dental \\ University, 1-5-45 Yushima, Bunkyo-ku, Tokyo 113-8549, Japan \\ ${ }^{2}$ Center of Excellence Program for Frontier Research on Molecular Destruction and Reconstruction of Tooth and Bone, \\ Tokyo Medical and Dental University, 1-5-45 Yushima, Bunkyo-ku, Tokyo 113-8549, Japan \\ ${ }^{3}$ Department of Restorative Dentistry, Division of Oral Health Sciences, Hokkaido University Graduate School of Dental \\ Medicine, North 13, West 7, Kita-ku, Sapporo 060-8586, Japan \\ Corresponding author, Yasushi SHIMADA; E-mail: shimada.ope@tmd.ac.jp
}

Received August 21, 2006 /Accepted October 20, 2006

To compare the pulpal responses to light-cured and self-cured resin cements, cervical cavities were prepared in monkey's teeth, followed by application of etching gel and adhesive (Single Bond). A dual-cure resin cement (RelyX ${ }^{\text {тм }}$ ARC) was applied, and hybrid composite inlays (Estenia) were bonded to the cavities. In one group, the cavities were photoirradiated for 20 seconds and the resin cement light-cured. In the other group, the resin cement was self-cured for six minutes without any photoirradiation. After experimental periods of seven, 28, and 70 days, histological features of pulp tissue were evaluated and compared. Results showed no significant differences in the histological features of the pulp tissues between the two curing methods. Both light-cured and self-cured resin cements showed acceptable biological compatibility with the monkey pulp. No bacterial penetration along the cavity walls was detected with either curing method.

Keywords: Pulpal response, Dual-cure resin cement, Composite resin inlay

\section{INTRODUCTION}

Advances in dental adhesives have led to the development of an indirect adhesive procedure for esthetic restorations. Tooth-colored inlays, bonded to the tooth structure using a resin cement, have fairly widespread clinical applications, such that they have become a viable and esthetic alternative to amalgam or metal inlays for posterior tooth restorations ${ }^{1-4)}$. Leveraging on the successful improvements in the adhesives for direct composite restorations, the bonding capacity of resin cements has also been improved by employing similar functional monomers used in the adhesives for direct composites. Formation of a hybrid layer that consists of a molecular-level mixture of adhesive polymers and hard dental tissues is recognized as one of the attributes that contributes to the high bond strength and minimum leakage of adhesive resin cements ${ }^{5}$.

Nonetheless, evidence of insufficient marginal sealing of bonded resin inlays has also been shown in both in vivo and in vitro studies, especially if resin cements were self-cured without any photoirradiation ${ }^{6)}$. While adhesively luted inlays are believed to reduce the side effects of polymerization contraction of composites over direct restorations, lower bond strength values of self-cured resin cements due to the lower degree of conversion of resinous materials have been reported $^{7-9}$. It has been demonstrated that the amount of unconverted monomers within the resinous materials is significantly influenced by the curing strategy used for the material.

Once these residual monomers reach the pulp tissue, a foreign body reaction may be elicited, causing some inflammatory effects ${ }^{10,11}$. Monomers like BisGMA, HEMA, UDMA, TEGDMA, initiators, and solvents have already been associated with cytotoxic effects on fibroblast and odontoblast-like cells ${ }^{12,13}$. Several reports have indicated that these components individually or in combination ultimately compromise the pulp healing process ${ }^{10,12,13)}$. Moreover, in deep cavity preparations, the high water content may prevent adequate polymerization of resinous materials, which in turn may release high levels of unconverted components into an adjacent aqueous phase that can diffuse through dentin to the pulp ${ }^{10,11}$. On the contrary, interactions between the different resinous materials were reported to be less toxic than the sum of the individual toxicities ${ }^{14}$. Additionally, although several in vitro studies have been developed for the testing of new adhesive materials, the complex morphological and physiological characteristics of dentin and pulp are yet to be duplicated in vitro ${ }^{15}$. For example, intrapulpal pressure could also reduce the cumulative diffusion of unconverted monomers through dentin ${ }^{16)}$.

The aim of this study, therefore, was to investigate the effects of the curing method of dual-cure resin cement on the reaction of monkey pulpal tissue after the bonding of tooth-colored resin inlays. In vivo bacterial leakage after the setting of resin inlays with two curing methods was also examined by means of histological staining of bacteria. 


\section{MATERIALS AND METHODS}

The treatment and use of animals in this study were approved by the Institutional Animal Care and Use Committee at Tokyo Medical and Dental University and Hokkaido University, in conformance to the protocol and facilities approved by the Committee on Ethical Guidelines for Animal Care of Tokyo Medical and Dental University. As for all the materials used in this study, they were handled according to the manufacturers' instructions.

\section{Preparation of composite resin inlays}

Resin inlays were prefabricated prior to cavity preparation, as described below. Impressions of approximately 2-mm end of diamond stones (ISO \# SB2, GC, Tokyo, Japan) were obtained using the wash technique with an addition silicone (Exafine, GC, Tokyo,

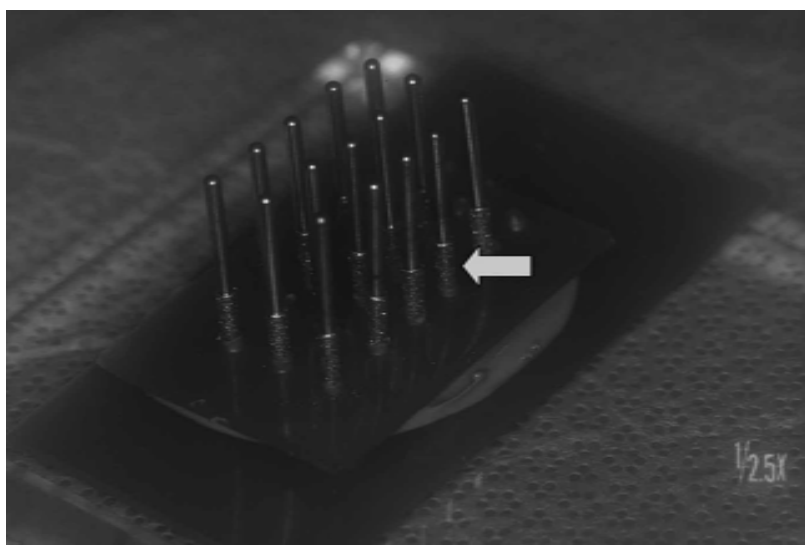

Fig. 1 2-mm end of \# SB2 diamond stones (arrow) were exposed through the paraffin wax sheet. Impressions were taken using an addition silicone material.

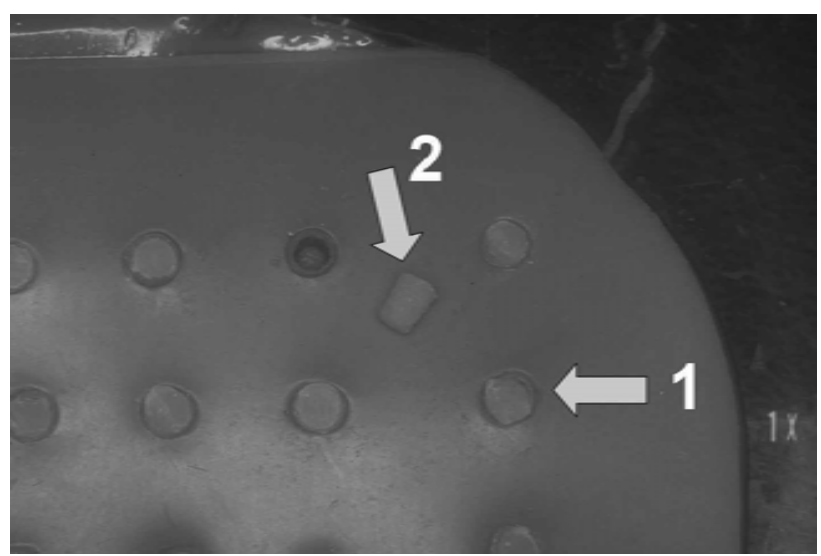

Fig. 2 Estenia was placed into the patterns of silicone impression (arrow 1) and cured with the combination of 60-second visible light photoirradiation and 110 ${ }^{\circ} \mathrm{C}$ heat for 15 minuets. Arrow 2: A cylindershaped resin inlay of $2-\mathrm{mm}$ height with the same size as the diamond stone.
Japan), which in turn were used as a mold for the composite resin inlays (Fig. 1). A resin composite for indirect restorations (Estenia, Shade DA2, Lot \# 00209B, Kuraray Medical Inc., Kurashiki, Japan) was placed into the impressions and irradiated for $60 \mathrm{sec}-$ onds using a visible light curing unit for the laboratory (a -Light II, J Morita Co., Tokyo, Japan), prior to heat curing at $110^{\circ} \mathrm{C}$ for 15 minutes in air (KL 100, Kuraray Medical Inc.) (Fig. 2). Very small cylinders of resin inlay, $2 \mathrm{~mm}$ high with the same size as the diamond stone, were removed from the impression (Fig. 2) and silanized with the application of a silane agent (Ceramic Primer, Lot \# 4WA, 3M ESPE, St. Paul, MN 55144-1000, USA) for five seconds.

\section{Cavity preparation and bonding procedure}

Two monkeys (Macaca fuscata, estimated age: 3-4 years, weight: 5-6 kg) were anesthetized by intramuscular injection of $2 \mathrm{mg} / \mathrm{kg}$ ketamine (Ketaral, Sankyo Co., Tokyo Japan) and intravenous injection of $2 \mathrm{mg} / \mathrm{kg}$ pentobarbital sodium (Nembutal Sodium Solution, Abbott Laboratories, Abbott Park, IL).

Cervical class $V$ cavities of approximately $2 \mathrm{~mm}$ in depth were prepared in the teeth using diamond stones (ISO \# SB2, GC) at high speed under water spray coolant. The cavosurface margin of the cavities was always in enamel.

The cavities were etched with phosphoric acid gel (Scotchbond etchant, Lot \# 4HP, 3M ESPE) for 15 seconds, followed by vigorous water spray for at least five seconds and airing for one second, keeping the cavity floor moist. Two coats of adhesive (Adper ${ }^{\text {TM }}$ Single Bond, Lot \# 4KE, 3M ESPE) were applied and air-thinned by a gentle stream of air for five seconds, followed by photoirradiation for 10 seconds. Dual-cure resin cement (RelyX ${ }^{\mathrm{TM}}$ ARC, Lot \# ELEU, 3M ESPE) was then handmixed and applied, and hybrid composite inlays (Estenia, Kuraray), which were prepared beforehand, were bonded to the cavities.

A total of 50 cavities were divided into two groups of 25 cavities each corresponding to the different curing strategies for the resin cement. In one group, light curing was carried out using a quartztungsten-halogen curing unit (Curing light XL3000, 3M ESPE) for 40 seconds, with a light output not less than $550 \mathrm{~mW} / \mathrm{cm}^{2}$ (light-cure group). In the other group, the resin cement was self-cured for six minutes without photoirradiation (self-cure group). After setting for 30 minutes, resin cement flash was removed with a high-speed superfine diamond bur (ISO \# V16ff, GC).

\section{Histological evaluation}

Experimental periods for the pulpal test were set at seven, 28, and 70 days to follow the ISO guidelines ${ }^{17}$. Curing methods and experimental periods were 
randomly assigned to each monkey's teeth, so that each experimental period included eight to nine teeth for each curing method. After the prescribed periods, the monkeys were sacrificed by an intravenous injection of $250 \mathrm{mg} / \mathrm{kg}$ of thiopental sodium (Ravonal, Tanabe Pharmaceutical Co., Osaka, Japan), and the teeth were removed from the jaws. Following fixation in phosphate-buffered solution of $4 \%$ formalin and $1 \%$ glutaraldehyde for one day, the teeth were decalcified with EDTA solution (Decalcifying Solution B, Wako Pure Chemical Industries Ltd., Osaka, Japan) at $4^{\circ} \mathrm{C}$ for four days, and then washed with running water for six hours. After removing the restorations, the teeth were dehydrated and finally embedded in paraffin.

Serial histological sections of $5 \mu \mathrm{m}$ thickness were prepared and mounted on charged slides. Hematoxylin and Eosin, and Brown \& Brenn gram stains were used. For all sections, four histological features (disorder of odontoblasts, inflammatory cell infiltration, reparative dentin formation, and bacterial staining) were evaluated. The evaluated features were then graded under a light microscope as None (0), Slight (1), Moderate (2), or Severe (3) ${ }^{18,19}$. Cavities that showed apparent pulpal exposure, namely two cavities in the self-curing group and three cavities in the light-cured group, were excluded from this study. The criteria are shown as follows:

\section{Disorder of odontoblasts}

None (0): Characterized by an absence of remarkable changes in the odontoblasts beneath cut dentinal tubules;

Slight (1): Characterized by a slight disarrangement of odontoblasts beneath cut dentinal tubules;

Moderate (2): Characterized by a disarrangement of most of the odontoblasts beneath cut dentinal tubules;

Severe (3): Characterized by a severe disarrangement or disappearance of odontoblasts beneath cut dentinal tubules.

\section{Inflammatory cell infiltration}

None (0): Characterized by an absence of inflammatory cells;

Slight (1): Characterized by the scattering of a small number of inflammatory cells;

Moderate (2): Characterized by a distinct increase in inflammatory cells;

Severe (3): Characterized by abscess formation in the pulp or pulpal necrosis.

\section{Reparative dentin formation}

None (0): No additional or abnormal increased thickness in circumpulpal dentin beneath cut dentinal tubules of cavity preparation;
Slight (1): A small thin rim of reparative dentin beneath cut dentinal tubules of cavity floor (thickness of the reparative dentin was less than twice the thickness of predentin);

Moderate (2): A moderate bulk of new reparative dentin beneath cut dentinal tubules of cavity floor (thickness of the reparative dentin was more than twice the thickness of predentin, but less than half that of pulpal thickness);

Severe (3): A large bulk of new reparative dentin beneath cut dentinal tubules of cavity floor (thickness of the reparative dentin was more than half that of pulpal thickness).

\section{Bacterial staining}

None (0): Absence of bacterial staining profiles throughout the section;

Slight (1): Positive bacterial staining profiles along the coronal wall or axial floor of cavity;

Moderate (2): Positive bacterial staining profiles within the cut dentinal tubules of axial floor;

Severe (3): Positive bacterial staining profiles within dental pulp.

Results pertaining to disorder of odontoblasts, inflammatory cell infiltration, reparative dentin formation, and bacterial staining were statistically analyzed using the Kruskal-Wallis test, with statistical significance defined as $\mathrm{p}<0.05$.

The remaining dentin thickness between the cavity floor and pulp (RDT) was also recorded, and variances in thickness were analyzed using one-way ANOVA $(\mathrm{p}<0.05)$.

\section{RESULTS}

Table 1 shows a summary of the findings in the histological sections and RDT. No statistically significant differences in RDT were found among the groups (one-way ANOVA, $\mathrm{F}=1.060, \mathrm{p}=0.3973$ ).

\section{Disorder of odontoblasts}

Moderate to severe disorders were detected with both curing methods for all experimental periods. In the self-cured group, one moderate and three slight disorders of eight specimens were seen at seven days; one severe, one moderate, and one slight disorder of six specimens were seen at 28 days; and one moderate and five slight reactions of nine specimens were seen at 70 days (Fig. 3).

The light-cured group showed one moderate and three slight disorders of seven specimens at seven days; two moderate and one slight disorder of seven specimens at 28 days; and one moderate and one slight reaction of eight specimens at 70 days (Fig. 4).

Statistical analysis showed no significant differences in odontoblastic disorder between the two curing methods in any of the experimental periods 
Table 1 Grading of histopathological features of serial sections.

\begin{tabular}{|c|c|c|c|c|c|c|c|c|c|c|c|c|c|}
\hline & & \multirow{2}{*}{$\mathrm{n}$} & \multirow{2}{*}{$\begin{array}{l}\text { RDT } \\
(\mathrm{mm})\end{array}$} & \multicolumn{5}{|c|}{ Disorder of odontoblasts } & \multicolumn{5}{|c|}{ Inflammatory cell infiltration } \\
\hline & & & & 0 & 1 & 2 & 3 & $\mathrm{Av}$ & 0 & 1 & 2 & 3 & $\mathrm{Av}$ \\
\hline \multirow{3}{*}{ Self-cure } & 7 days & 8 & 0.49 & 4 & 3 & 1 & 0 & 0.63 & 8 & 0 & 0 & 0 & 0 \\
\hline & 28 days & 6 & 0.57 & 3 & 1 & 1 & 1 & 1 & 4 & 1 & 2 & 0 & 0.83 \\
\hline & 70 days & 9 & 0.31 & 3 & 5 & 1 & 0 & 0.78 & 9 & 0 & 0 & 0 & 0 \\
\hline \multirow{5}{*}{ Light-cure } & 7 days & 7 & 0.41 & 3 & 3 & 1 & 0 & 0.71 & 7 & 0 & 0 & 0 & 0 \\
\hline & 28 days & 7 & 0.42 & 4 & 1 & 2 & 0 & 0.71 & 6 & 0 & 1 & 0 & 0.29 \\
\hline & 70 days & 8 & 0.26 & 6 & 1 & 1 & 0 & 0.38 & 8 & 0 & 0 & 0 & 0 \\
\hline & & & RDT & \multicolumn{5}{|c|}{ Reparative dentin formation } & \multicolumn{5}{|c|}{ Bacterial staining } \\
\hline & & $\mathrm{n}$ & $(\mathrm{mm})$ & 0 & 1 & 2 & 3 & $\mathrm{Av}$ & 0 & 1 & 2 & 3 & $\mathrm{Av}$ \\
\hline \multirow{3}{*}{ Self-cure } & 7 days & 8 & 0.49 & 8 & 0 & 0 & 0 & 0 & 8 & 0 & 0 & 0 & 0 \\
\hline & 28 days & 6 & 0.57 & 4 & 1 & 1 & 0 & 0.5 & 6 & 0 & 0 & 0 & 0 \\
\hline & 70 days & 9 & 0.31 & 5 & 1 & 3 & 0 & 0.78 & 9 & 0 & 0 & 0 & 0 \\
\hline \multirow{3}{*}{ Light-cure } & 7 days & 7 & 0.41 & 7 & 0 & 0 & 0 & 0 & 7 & 0 & 0 & 0 & 0 \\
\hline & 28 days & 7 & 0.42 & 6 & 0 & 1 & 0 & 0.29 & 7 & 0 & 0 & 0 & 0 \\
\hline & 70 days & 8 & 0.26 & 6 & 1 & 1 & 0 & 0.38 & 8 & 0 & 0 & 0 & 0 \\
\hline
\end{tabular}

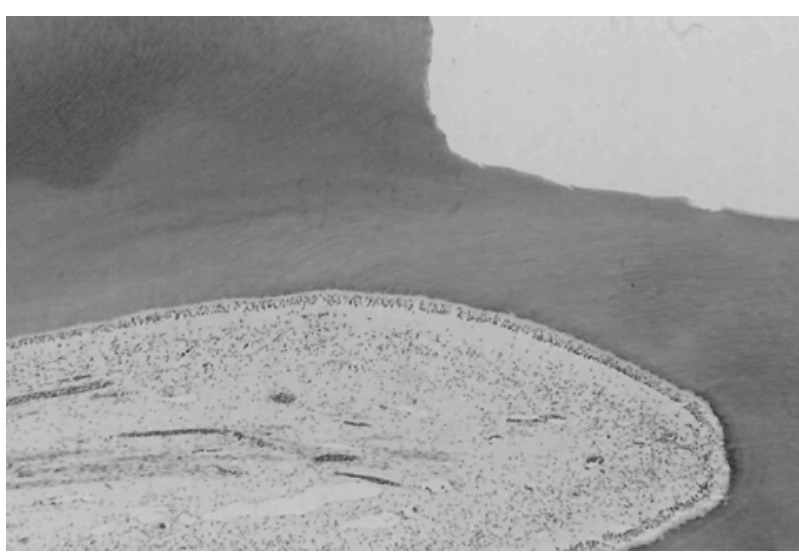

Fig. 3 Self-cure at 7 days. Remaining dentin thickness was approximately $0.45 \mathrm{~mm}$. Slight level of odontoblastic disorder beneath cut dentinal tubules was observed. No inflammatory cell infiltration was seen (magnification $\times 40$ ).

(Kruskal-Wallis test, $\mathrm{p}=0.8795$ ).

\section{Inflammatory cell infiltration}

Inflammatory cell infiltration was seen only at 28 days. Two moderate and one slight reaction of six specimens were detected in the self-cured group, and one moderate reaction was seen in the light-cured group (Fig. 5).

Statistical analysis showed no significant differences in inflammatory cell infiltration between the two curing methods in any of the experimental periods (Kruskal-Wallis test, $\mathrm{p}=0.5994$ ).

\section{Reparative dentin formation}

At seven days in both test groups, there were no indications of any reparative dentin beneath the cut tubules of the remaining dentin.

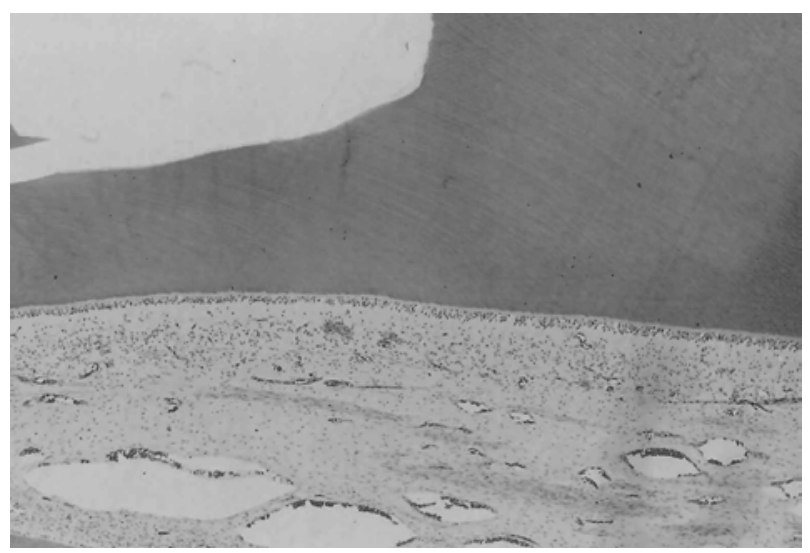

Fig. 4 Light-cure at 7 days. Remaining dentin thickness was approximately $0.4 \mathrm{~mm}$. Slight level of odontoblastic disorder beneath cut dentinal tubules was seen. No inflammatory cell infiltration was observed (magnification $\times 40$ ).

The self-cured group showed one case of moderate and one case of slight dentin formation of six specimens at 28 days, and three cases of moderate and one case of slight formation of nine specimens at 70 days (Figs. 5 and 6). In the light-cured group, one case of moderate formation of seven specimens was seen at 28 days, and one case of moderate and one case of slight formation of eight specimens were seen at 70 days (Fig. 7).

Statistical analysis showed no significant differences in reparative dentin formation between the two curing methods in any of the experimental periods (Kruskal-Wallis test, $\mathrm{p}=0.5805$ ).

\section{Bacterial staining}

Bacterial penetration along the cavity walls could not be detected in any of the experimental groups. 


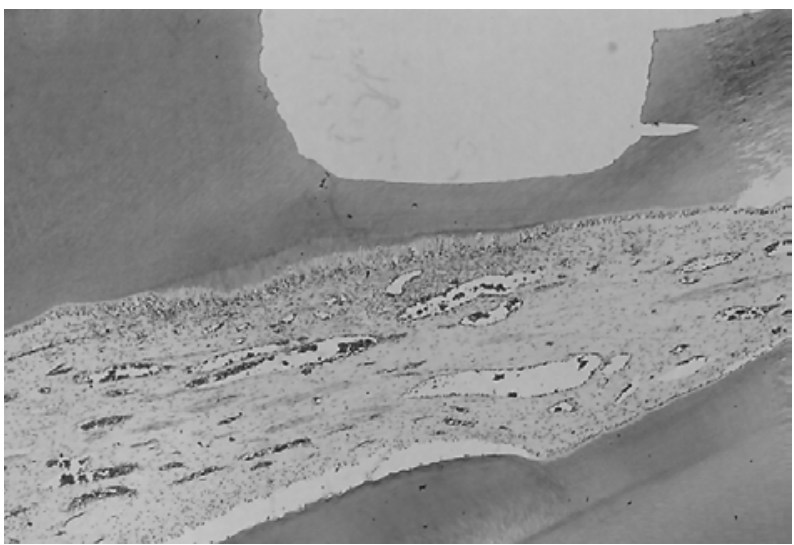

Fig. 5 Self-cure at 28 days. Remaining dentin thickness of this specimen was thin (approximately 0.15 $\mathrm{mm})$. Moderate level of inflammatory cell infiltration was observed. Odontoblastic disorder was slight and reparative dentin formation was moderate (magnification $\times 40$ ).

\section{DISCUSSION}

This in vivo study evaluated the effects of two curing strategies of a dual-cure resin cement on pulpal irritation level toward resin inlay restorations using monkey pulp. Owing to the limitations and difficulties of animal treatment, resin inlays were fabricated in advance and set immediately after cavity preparation. Impression or provisional restoration could be omitted in this process due to the employment of standardized cavity preparation and standard-sized resin inlays ${ }^{19}$. Although a few points of discrepancy exist from the clinical steps for indirect restorations, a previous in vivo study indicated slight pulpal irritation resulting from impression and temporization ${ }^{20}$. This is because a provisional restoration helps to heal pulpal damage resulting from cavity preparation, and therefore irritation levels following the clinical steps may be lower.

Results of this study indicated that both lightcuring and self-curing methods of resin cement induced slight to moderate levels of odontoblastic disorders in the pulp after a seven-day test interval. At seven days, since similar irritation levels of odontoblastic disorders without any inflammatory cell infiltration were observed in both curing methods, these reactions seemed to arise from the deep cavity preparation or bonding process for the setting of resin inlay. It is widely recognized that pulpal irritation levels of cavity preparation increase if the remaining thickness of cavity floor dentin is thin ${ }^{18-21)}$. In the present study, cavity depth was controlled to approximately $2 \mathrm{~mm}$. Employing this standardized cavity preparation, the remaining dentin thickness (RDT) of three-day specimens ranged from 0.20 to $0.85 \mathrm{~mm}$. While a few specimens with extremely deep cavity preparation showed moderate levels of

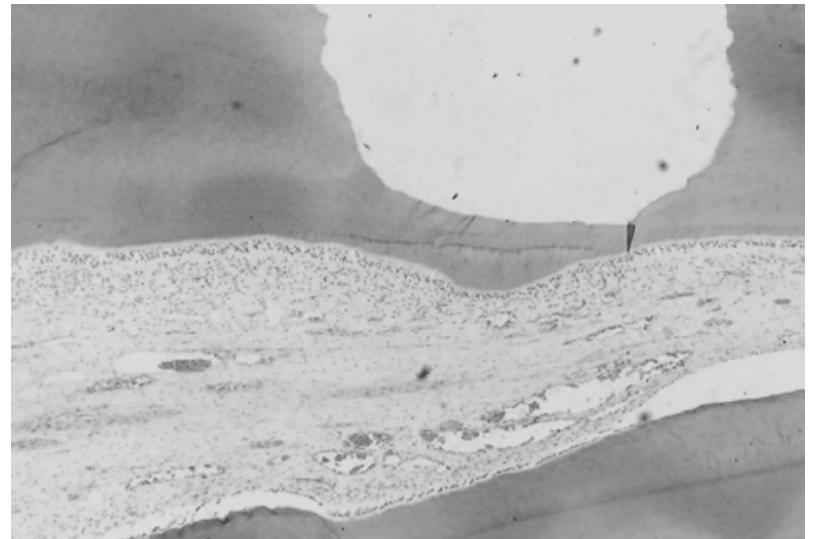

Fig. 6 Self-cure at 70 days. Remaining dentin thickness of this specimen was very thin (approximately $0.07 \mathrm{~mm}$ ). However, no inflammatory cell infiltration was seen. Odontoblastic layer was slightly disordered and reparative dentin deposits were at moderate level (magnification $\times 40$ ).

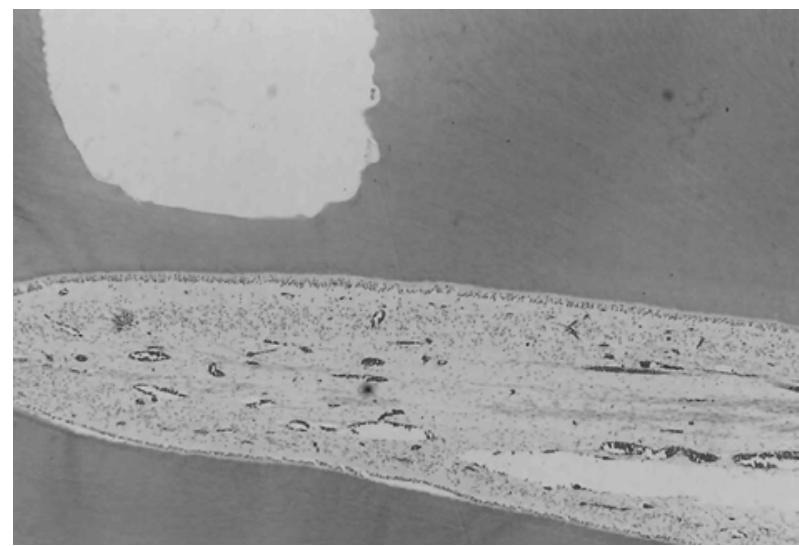

Fig. 7 Light-cure at 70 days. Remaining dentin thickness was also thin in this specimen (approximately $0.18 \mathrm{~mm}$ ). No inflammatory cell infiltration nor reparative dentin formation could be seen (magnification $\times$ 40).

disarrangement, the correlation between RDT and odontoblastic disorders could not be detected due to the controlled cavity depth.

In this study, phosphoric acid etching gel attached to the resin cement was applied to the cavity wall to remove the smear layer. Several studies have shown that total-etched dentin by phosphoric acid sometimes enhanced the reduction of odontoblasts more than nonetched cavities at the initial stage, but these differences did not persist over the long term ${ }^{18}$. After etching and rinsing, we kept the etched dentin moist prior to application of the adhesive, following the instructions for this material. Since the observed odontoblastic changes with the two curing methods were only mild to slight with lower degree of odontoblastic reduction, the wet bond method could indeed reduce the irritation level of the etching process. Meanwhile, the effects of air-drying on odontoblastic changes have been discussed, especially 
after total etching, and this mild level of reaction appeared to be due to the less air-drying ${ }^{18}$.

At 28 days, the light-cured group showed one moderate reaction of inflammatory cell infiltration. At the same time, one slight and two moderate reactions were observed in the self-cured group. Although no significant differences in inflammatory cell infiltration were detected between the two curing methods, the self-cured specimen showed a slightly higher number of inflammatory reaction cases. It is well known that the conversion of monomers to polymers is never complete, regardless of the type of activation $^{7,13,22-24)}$. Unreacted resinous materials like Bis-GMA, HEMA, UDMA, TEGDMA, initiators, and solvents leach out from the material and have been shown to cause cytotoxic reactions in in vitro studies $^{10-12}$. Since light irradiation can achieve a relatively higher conversion ratio of resin cements over the self-cure method, pulpal irritation due to unconverted resinous materials might be lower ${ }^{7,14-16)}$. However, these cases of inflammatory cell infiltration observed at 28 days disappeared at 70 days for both curing methods. Several reports of in vitro studies have indicated that the release of unconverted components into an adjacent aqueous phase was at its highest during the initial immersion, with levels decreasing thereafter ${ }^{10,11,14)}$. Accordingly then, this result seemed to indicate that the irritation levels due to unconverted products from the cement were low, even when it was self-cured.

In this study, prior to the application of Rely $\mathrm{X}^{\mathrm{TM}}$ ARC cement, total-etch adhesive was applied to the cavity wall and light-cured, creating a resin-tooth hybridized zone with an approximate depth of 5 to $10 \mu \mathrm{m}^{5,25)}$. Since the sealing effect of this hybridized zone has been shown in a series of studies, it could be said that this zone blocked the diffusion of unreacted resinous materials to the pulp, resulting in lower levels of pulpal reaction with both self-cure and light-cure methods ${ }^{5,6,20)}$.

It is noteworthy that odontoblastic disorders remained in both self-cured and light-cured specimens even after 28- or 70-day test intervals. Since traumatic effects due to cavity preparation should have largely disappeared by the intermediate ISOstipulated time interval, these remaining odontoblastic disorders could be an indication of some stimulant effect from the restoration itself ${ }^{15,17-20)}$. Although the reason for this remaining disorder was still unclear, unconverted resinous materials within the cement might in part induce this reaction. It is well documented that, after dental injury and irreversible odontoblastic damage, dental pulp is able to produce a reparative dentin matrix - which is secreted by a secondary generation of odontoblast-like cells ${ }^{26)}$. On this note, our results were in agreement with the previous report ${ }^{26)}$ : whereby slight to moderate levels of reparative dentin formation were found at the 70day test interval. Consequently, it was highly probable that this remaining disorder indicated a remodeling of odontoblasts and that the healing process still continued at 28 and 70 days. Indeed, the relatively lower level of disorders observed in the 70day light-cured specimens compared with the 28-day specimens seemed to support this speculation.

Despite rapid progress in the advancement of adhesive materials coupled with high bond strength rendered by recently developed resinous materials, microleakage along restorative sites remains a significant challenge in bonded inlays $s^{6,7,27)}$. However, in this in vivo study, no bacterial penetration was detected the class $\mathrm{V}$ cavity wall for up to 70 days, when the resin inlays were bonded with RelyX ${ }^{\mathrm{Tm}}$ ARC. In light of this result, we could conclude that the bonding performance of this resin cement was excellent for both self-cure and light-cure methods. It should also be mentioned that the adhesive employed in this resin cement seemed to contribute to this ideal result regarding sealing capacity ${ }^{25,28)}$.

In view of previous adhesion results obtained from bonded resin inlays to dentin using self-cured and light-cured resin cements, it was of interest for us to determine the bacterial leakage behavior of this material ${ }^{8,9}$. Nonetheless, it is common knowledge that adhesively luted resin inlays significantly reduce the side effects of polymerization stress of resin composites, and that the bonding capacity of self-cured resin cements is generally inferior to that of lightcured cements, ${ }^{2,3,8,9,22-24)}$. Within the limitations of this in vivo study, self-cured Rely $\mathrm{X}^{\mathrm{TM}} \mathrm{ARC}$ could bond the resin inlay up to 70 days without bacterial penetration. At this juncture, it should be put into perspective that the curing process of resin cements is a complicated one. During the transition from liquid to viscoelastic phase, the resin cement can be used without inducing damage to the internal structures of both the cement and adhesive ${ }^{27}$. Since this soft and flowable stage continues longer in the self-cure method, movement of resin cement during shrinkage might compensate for the shrinkage, thus resulting in an unexpected but excellent marginal seal.

The results of this present study were limited to biocompatibility and in vivo microleakage in monkey teeth only. Therefore, it was deemed both expedient and necessary to carry out further bonding durability tests under occlusal stress as well as more detailed clinical evaluations of the resin cement, Rely $\mathrm{X}^{\mathrm{TM}}$ ARC.

\section{ACKNOWLEDGEMENTS}

This work was supported by a grant for the Center of Excellence Program for Frontier Research on Molecular Destruction and Reconstruction of Tooth 
and Bone at Tokyo Medical and Dental University.

\section{REFERENCES}

1) Burrow MF, Nikaido T, Satoh M, Tagami J. Early bonding of resin cements to dentin - Effect of bonding environment. Oper Dent 1996; 21:196-202.

2) El-Mowafy OM, Rubo MH, El-Badrawy WA. Hardening of new resin cements cured through a ceramic inlay. Oper Dent 1999; 24:38-44.

3) Foxton RM, Pereira PN, Nakajima M, Tagami J, Miura H. Durability of the dual-cure resin cement/ ceramic bond with different curing strategies. J Adhes Dent 2002; 4:49-59.

4) Shimada Y, Yamaguchi S, Tagami J. Micro-shear bond strength of dual-cured resin cement to glass ceramics. Dent Mater 2002; 18:380-388.

5) Nakabayashi N, Kojima K, Masuhara E. The promotion of adhesion by the infiltration of monomers into tooth substrates. Journal Biomed Mater Res 1982; 16:265-273.

6) Jayasooriya PR, Pereira PNR, Nikaido T, Burrow MF, Tagami J. The effect of a "resin coating" on the interfacial adaptation of composite inlays. Oper Dent 2003; 28:28-35.

7) Silikas N, Eliades G, Watts DC. Light intensity effects on resin-composite degree of conversion and shrinkage strain. Dent Mater 2000; 16:292-296.

8) Shimada Y, Tomimatsu N, Suzuki T, Uzzaman MA, Burrow MF, Tagami J. Shear bond strength of tooth-colored indirect restorations bonded to coronal and cervical enamel. Oper Dent 2005; 30(4):468-473.

9) Shimada Y, Sattabanasuk V, Sadr A, Yuan Y, He Z, Tagami J. Shear bond strength of tooth-colored indirect restorations bonded to mid-coronal and cervical dentin. Dent Mater J 2006; 25(1):7-12.

10) De Souza Costa CA, Hebling J, Randall RC. Human pulp response to resin cements used to bond inlay restorations. Dent Mater 2006; 22:954-962.

11) Hamid A, Hume WR. A study of component release from resin pit and fissure sealants in vitro. Dent Mater 1997; 13:98-102.

12) Geurtsen W, Lehmann F, Spahl W, Leyhausen G. Cytotoxicity of 35 dental resin composite monomers/ additives in permanent 3T3 and three human primary fibroblast cultures. J Biomed Mater Res 1998; 41:474480.

13) Sideridou ID, Achilias DS. Elution study of unreacted Bis-GMA, TEGDMA, UDMA, and Bis-EMA from light-cured dental resins and resin composites using HPLC. J Biomed Mater Res 2005; 74:617-626.

14) Ratanasathien S, Wataha JC, Hanks CT, Dennison
JB. Cytotoxic interactive effects of dentin bonding components on mouse fibroblasts. J Dent Res 1995; 74:1602-1606.

15) Cox CF. Pulp protection and direct capping with $\mathrm{Ca}(\mathrm{OH})_{2}$ versus adhesive resin systems: a review of factors leading to failure or success. In: Proceedings of 3rd International Kuraray Symposium on Advanced Adhesive dentistry, Tagami J, Toledano M, Prati C (eds.), Kuraray Co. Ltd., Tokyo, 1999, pp.149175.

16) Gerzina TM, Hume WR. Effect of hydrostatic pressure on the diffusion of monomers through dentin in vitro. J Dent Res 1995; 74: 369-373.

17) EN ISO 7405:1997. Dentistry: Preclinical evaluation of biocompatibility of medical devices used in dentistry. Test methods for dental materials.

18) Shimada Y. Pulpal response to various dentin conditioning agents. Japan Journal of Conservative Dentistry 1992; 35:69-100.

19) Uzzaman MA, Shimada Y, Seki Y, Tagami J. Pulpal response to a hybrid composite resin inlay bonded with a newly developed resin-modified glass ionomer luting cement. Dent Mater J 2005; 24(2):178-186.

20) Inokoshi S, Shimada Y, Fujitani M, Otsuki M, Shono T, Onoe N, Morigami M, Takatsu T. Monkey pulpal response to adhesively luted indirect resin composite inlays. Oper Dent 1995; 20:111-118.

21) Cox CF, Keall CL, Keall HJ, Ostro E, Bergenholtz G. Biocompatibility of surface-sealed dental materials against exposed pulps. J Prosthet Dent 1987; 57:1-8.

22) Aksornmuang J, Nakajima M, Foxton RM, Tagami J. Regional bond strength of four self-etching primer/ adhesive systems to root canal dentin. Dent Mater $\mathrm{J}$ 2005; 24:261-267.

23) Ikeda M, Nikaido T, Foxton RM, Tagami J. Shear Bond strengths of indirect resin composites to hybridceramic. Dent Mater J 2005; 24:238-243.

24) Yamauti M, Nikaido T, Ikeda M, Otsuki M, Tagami J. Microhardness and Young's modulus of a bonding resin cured with different curing units. Dent Mater J 2004; 23:457-466.

25) Shimada Y, Iwamoto N, Kawashima M, Burrow MF, Tagami J. Shear bond strength of current adhesive systems to enamel, dentin and dentin-enamel junction region. Oper Dent 2003; 28:585-590.

26) Smith AJ. Pulpal responses to caries and dental repair. Caries Research 2002; 36:223-232.

27) De Jager N, Pallav P, Feilzer AJ. Finite element analysis model to simulate the behavior of luting cements during setting. Dent Mater 2005; 21:1025-1032.

28) Shimada Y, Tagami J. Effects of regional enamel and prism orientation on resin bonding. Oper Dent 2003; 28:20-27. 